Hispania, LXIII/2, núm. 214(2003)

\title{
ESPACIOS Y FORMAS DE LA SOCIABILIDAD EN LA ESPAÑA CONTEMPORÁNEA
}

\author{
INTRODUCCIÓN
}

La «sociabilidad» es ya una categoría histórica relativamente asentada en la historiografía española contemporaneísta desde al menos una década, especialmente en los estudios locales, a partir del número pionero de la desgraciadamente ya desaparecida revista Estudios de Historia Social dedicado a «La sociabilidad en la España contemporánea» ${ }^{1}$. La bibliografía acerca de la sociabilidad empieza a ser en efecto realmente consecuente: numerosas monografías, más o menos explicativas o descriptivas, se han publicado hasta la fecha, algunos coloquios se han celebrado ya sobre el tema, empezando por el coloquio celebrado en la Casa de Velázquez en 1987 y siguiendo con los de Besançon, Ciudad Real, Valenciennes y Valladolid ${ }^{2}$. Disponemos incluso de

1 Estudios de Historia Social, Madrid, no 50-51, Julio-diciembre 1989, pp. 131-305 (BREY, Gérard: «Aproximación a la sociabilidad popular en las ciudades gallegas (1833-1914)», pp. 223242; GUEREÑA, Jean-Louis: «Fuentes para la historia de la sociabilidad en la España contemporánea», pp. 273-305, y «Una aproximación a la sociabilidad popular: el caso de Asturias bajo la Restauración (1875-1900)», pp. 201-222; LÉCUYER, Marie-Claude: «Algunos aspectos de la sociabilidad en España hacia 1840», pp. 145-159; MAURICE, Jacques: «Propuestas para una historia de la sociabilidad en la España contemporánea», pp. 133-143; MORALES, Manuel: «La sociabilidad popular en Málaga, 18401874: de la tutela burguesa a la afirmación de una identidad diferenciada», pp. 243-271; RALLE, Michel: «La sociabilidad obrera en la sociedad de la Restauración (1875-1910)», pp. 161-199). Ver GUEREÑA, Jean-Louis: «Hacia una historia socio-cultural de las clases populares en España (18401920)», Historia Social, Valencia, n 11, Otoño de 1991, pp. 149-151.

2 GuereÑA, Jean-Louis y TianA Ferrer Alejandro (Eds.): Clases populares, Cultura, Educación. Siglos XIX-XX, Madrid, Casa de Velázquez-U.N.E.D., 1989, pp. $377-453$ («Formas y lugares de la sociabilidad popular»); CARRASCO, Raphaël (Ed.): Solidarités et sociabilités en Espagne (XVI $-X X^{e}$ siècles), Paris, Les Belles Lettres (Annales Littéraires de l'Université de Besançon, vol. 436), 1991, 404 p.; SÁNCHEZ SÁNCHEZ, Isidro y VILLENA ESPINOSA, Rafael (Eds.): Sociabilidad fin de siglo. Espacios asociativos en torno a 1898, Cuenca, Ediciones de la Universidad de Castilla-La Mancha, 1999, 326 p.;

Hispania, LXIII/2, núm. 214 (2003) 409-414 
varios balances historiográficos, a cargo de Jordi Canal por ejemplo ${ }^{3}$, y de intentos de revisión metodológica ${ }^{4}$, a los cuales remitimos naturalmente, sin olvidar la existencia de varios grupos de investigación dedicados al estudio de la sociabilidad, entre los cuales apuntemos el GEAS [Grupo de Estudios de Asociacionismo y Sociabilidad], afincado en la Universidad de Castilla-La Mancha $^{5}$. En las notas a los diferentes trabajos incluidos a continuación se podrán hallar de todos modos numerosas referencias bibliográficas a los estudios más representativos en cada ámbito de la sociabilidad.

No se trataba pues, en esta sección monográfica de Hispania, de añadir otro nuevo balance historiográfico general a los ya existentes y de demostrar, de paso, la extraordinaria vitalidad de los estudios en el campo de la sociabilidad, sino de intentar ofrecer al lector una serie de estudios sintéticos, a cargo de algunos de los más destacados especialistas, de algunos espacios específicos de sociabilidad (sea formal o informal, burguesa o popular, masculina o femenina...) con la finalidad de llegar en cierto modo a disponer de una «tipología» y de una cronología comparadas de algunos «modelos» de sociabilidad sobre la base de las monografías realizadas a escala local y de los propios trabajos de cada uno de los contribuyentes a esta sección.

No presentamos por lo tanto aquí una historia completa de los espacios y de las formas de sociabilidad en la España contemporánea, imposible por otra parte de llevar actualmente a cabo, sino un intento, que nos parece significativo, de reflexión plural sobre algunos escenarios privilegiados de sociabilidad. Nos parece en efecto que el conjunto de los estudios reunidos permite rastrear suficientemente la fecundidad y la operatividad de la noción de sociabilidad en el campo histórico, incluso si no están abordados todos los espacios y todas las formas de la sociabilidad.

No hay; además, fronteras estancadas entre las diferentes contribuciones, y el lector podrá hallar numerosas correspondencias entre todas ellas, como la importancia - y a veces la reivindicación - de la noción de «espacio" para que pueda realizarse o estructurarse mínimamente un ámbito de sociabilidad o la «multifuncionalidad» de los espacios y formas de sociabilidad que aparece en casi todos los trabajos. Junto a algunas estructuras de sociabilidad ya bien conocidas y estudiadas como el ateneo o el casino, se presentan otros espacios

\footnotetext{
MARTín Luis P. y BRenot Anne M. (Eds.): Les sociabilités dans le monde hispanique (XVIII ${ }^{\text {ime }}-\mathrm{XX}^{\mathrm{ime}}$ siècles). Formes, lieux et représentations, Valenciennes, Presses Universitaires de Valenciennes (Recherches Valenciennes 3), 2000, 319 p.; MAZA ZORRILlA, Elena (Ed.): Sociabilidad en la España contemporánea. Historiografía y problemas metodológicos, Valladolid, Universidad de Valladolid, 2002, 219 p.

3 CANAL, Jordi: «La sociabilidad en los estudios sobre la España contemporánea: una revisión", en MAZA ZORRILLA, Elena (Ed.): Sociabilidad en la España contemporánea, op. cit., pp. 35-55.

4 VAlín, Alberto (Ed.): La sociabilidad en la Historia contemporánea. Reflexiones teóricas y ejercicios de análisis, Ourense, Duen de Bux (Historia. Estudios -1), 2001, 236 p.

5 Grupo de Estudios de Asociacionismo y Sociabilidad: España en sociedad. Las asociaciones a finales del siglo XIX, Cuenca, Ediciones de la Universidad de Castilla-La Mancha, 1998, 229 p.
} 
menos abordados, por lo menos desde la perspectiva de la sociabilidad, como el burdel, el club deportivo o la taberna.

La cronología y la geografía enfocadas por las contribuciones -en principio el conjunto de la España de los siglos XIX y XX - pueden ser algo distintas entre los diversos trabajos, sin que ello entorpezca la necesaria mirada comparativa. Como es lógico, los autores han privilegiado las zonas y las épocas de sus propios estudios y las que cuentan con mayor número de monografías. En todo caso, el papel y la importancia de la Ley de Asociaciones de 1887 pueden constatarse en casi todas las contribuciones.

La distinción que operara antaño Maurice Agulhon entre las dimensiones más o menos formalizadas de la sociabilidad, si bien conserva parte de su pertinencia, merece matizarse un tanto. Y la tertulia, ese fenómeno tan inserto en las prácticas hispanas de sociabilidad ${ }^{6}$, aparece en particular a menudo en los distintos espacios abordados. Las dimensiones implícitas de sociabilidad aparecen por lo tanto más importantes dentro de esta perspectiva que las orientaciones explícitas de una u otra estructura.

Antes de pasar a abordar los distintos espacios y formas estudiadas en esta sección, no nos parece inútil recordar la aparición y el significado general de la noción de sociabilidad.

El término de «sociabilidad» es una vieja palabra, ya presente en el léxico castellano desde al menos mediados del siglo XVIII. Aparece por ejemplo en el Diccionario de Autoridades (1739) como «el tratamiento y correspondencia de unas personas con otras», y en el Diccionario castellano con las voces de Ciencias y Artes y sus correspondientes en las tres lenguas, francesa, latina e italiana de Esteban de Terreros y Pando (1776-1793), como «sociedad, trato de unas personas con otras». En el siglo XIX, numerosas serán las obras en valerse de las voces «sociabilidad» $\mathrm{y}$ «sociable» al referirse a las relaciones humanas.

En el campo de las ciencias sociales, y ya en el siglo XX, fue utilizada primero en el campo sociológico (como otras tantas nociones manejadas en Historia, y particularmente en Historia social), destacándose al respecto la obra de Georges Gurvitch ${ }^{7}$, e irrumpe en el campo histórico en 1966 de manos del historiador francés Maurice Agulhon, profesor de Historia contemporánea de 1986 a 1998 en el Colegio de Francia en París, con su estudio acerca de las

6 Ver Tierno GalváN, Enrique: «Notas sobre la tertulia», recogidas en sus Escritos (19501960), Madrid, Editorial Tecnos, 1971, y en la recopilación Desde el espectáculo a la trivialización, Madrid, Editorial Tecnos, 1987, pp. 241-266.

7 Gurvitch, Georges: La vocation actuelle de la sociologie, Paris, Presses Universitaires de France, 1963 ( $1^{a}$ ed., 1950), pp. 119-248 («L'échelle microsociologique: manifestations de la sociabilité»). Ver también del mismo autor sus artículos anteriores sobre la sociabilidad: «Analyse critique de quelques classifications des formes de la sociabilité», Archives de philosophie du droit et de sociologie juridique, Paris, $\mathrm{n}^{\circ} 3-4,1935$, pp. 43-91, y «Essai d'une classification pluraliste des formes de la sociabilité", Annales sociologiques, Paris, 1937, pp. 1-48.

Hispania, LXIII/2, núm. 214 (2003) 409-414 
cofradías meridionales dieciochescas ${ }^{8}$. Según su propio testimonio9, Agulhon recogía entonces la noción del erudito provenzal Fernand Benoit ${ }^{10}$. Y la sociabilidad conoció posteriormente y muy rápidamente un éxito considerable -no carente por supuesto de cierto efecto de moda - en la historiografía occidental, en particular la francesa, la italiana y la española.

En varios trabajos, algo sorprendido sin duda y perplejo ante la multiplicación de estudios referentes a la sociabilidad y que hacían explícita referencia a sus propios trabajos, Maurice Agulhon ha intentado realizar alguna auto-crítica sobre su investigación pionera, precisar y perfilar cada vez más el uso histórico de la noción, planteándose la validez y el campo de esta ya categoría histórica, que forma ahora plenamente parte de las herramientas metodológicas de los historiadores.

En particular, la identificación entre sociabilidad y «temperamento meridional» que Agulhon introdujo en su primer trabajo de 1966 tras sus lecturas de Fernand Benoit, no podía resistir mucho tiempo al análisis histórico. Pero al mismo tiempo fue "como quien dice, un rayo de luz, y la ocasión de abrir, con algunas falsas pistas, la vía de algunas investigaciones novedosas»"11. Antoine Prost se refiere así al verdadero «choque» que los historiadores de su generación experimentaron tras la lectura de La sociabilidad meridional —reeditada en 1968 bajo el título de Penitentes y Masones $^{12}$ - y a las nuevas dimensiones introducidas entonces en la investigación histórica, en particular en el campo de la historia económica y social ${ }^{13}$.

¿Cómo definir pues la sociabilidad como categoría histórica? De una acepción limitada y reductora, la noción ha evolucionado progresivamente hacia una concepción abierta, integrando a las veces los aspectos más formalizados (la vida asociativa) y los menos estructurados de la vida cotidiana.

8 AGUlHon, Maurice: La sociabilité méridionale (Confréries et associations dans la vie collective en Provence orientale à la fin du XVIII siècle), Aix-en-Provence, Éd. La Pensée Universitaire, 1966, 2 vols., 878 p. Se trataba de una tesis de doctorado (llamada entonces de «tercer ciclo»). Ver también su primera aproximación al tema en "Les associations (Confréries religieuses et loges maçonniques) en Provence orientale à la fin de l'Ancien Régime», en Actes $d u 84^{\circ}$ Congrès National des Sociétés Savantes. Poitiers 1962, Paris, Imprimerie Nationale, 1963, pp. 73-86.

9 Agulhon, Maurice: «Vu des coulisses», en NORA Pierre (Ed.): Essais d'ego-histoire, Paris, Gallimard (Bibliothèque des histoires), 1987, p. 33.

10 Benoît, Fernand: La Provence et le Comtat Venaissin, Paris, Gallimard («Les provinces françaises"), 1949, p. 25.

11 AGULHON, Maurice: «Les chocs d'une vie d'historien», en La bibliothèque imaginaire $d u$ Collège de France, Paris, Le Monde Editions, 1990, p. 13.

12 Agulhon, Maurice: Pénitents et Francs-Maçons de lancienne-Provence. Essai sur la sociabilité méridionale, Paris, Fayard, 1968, 452 p. Como se puede notar, por prudencia, el término de «sociabilidad» quedaba relegado en el subtítulo del trabajo.

13 PROST, Antoine: «Sociale et.culturelle indissociablement»; en Rioux, Jean-Pierre y Sirinelli, Jean-François (Eds.): Pour une Histoire culturelle, Paris, Éditions du Seuil, 1997, p. 136.

Hispania, LXIII/2, núm. 214 (2003) 409-414 
En un texto corto y relativamente poco conocido, el propio Agulhon nos ha ofrecido una definición relativamente concisa que consideramos como la más global e integradora de diversas formas de sociabilidad, entendiendo por tal «los sistemas de relaciones que relacionan a los individuos entre sí o que les reunen en grupos, más o menos naturales, más o menos forzosos, más o menos estables, más o menos numerosos ${ }^{14}$. Unos años más tarde, insistirá en esta cuestión de la formalización de la sociabilidad, en la aptitud a constituir y consolidar grupos humanos ${ }^{15}$.

La noción histórica de sociabilidad, citada y utilizada a menudo sin real reflexión sobre su contenido y problemática ha venido a ser víctima en algún modo de su éxito mismo. Pero nos parece seguir siendo válida, operativa y fecunda, siempre que se defina y limite un tanto su espacio, so pena de volverse un cajón de sastre y, por consiguiente, una noción inútil para la explicación histórica por demasiado ambigua. En efecto, podríamos decir sencillamente que si todo es sociabilidad, la sociabilidad no es nada y que no sirve para la explicación histórica.

La sociabilidad remite pues en la historiografía actual a la aptitud de los hombres para relacionarse en colectivos más o menos estables, más o menos numerosos, y a las formas, ámbitos y manifestaciones de vida colectiva que se estructuran con este objetivo. Una noción amplia desde luego pero indudablemente fecunda, en la encrucijada de la antropología cultural, la etnología de la vida cotidiana, la sociología del ocio y la historia social, política y cultural, y que supone pues multiplicidad de espacios y de formas.

La articulación dentro de la historia social puede facilitarnos un nuevo modo de abordar y relacionar cuestiones antiguas y de plantear nuevos objetos históricos bajo una misma mirada integradora, como, en particular, la historia del ocio, del tiempo libre o de las fiestas ${ }^{16}$, permitiendo entender la estructuración de identidades colectivas y la noción de redes a nivel local. Por otra parte, la perspectiva del género ha de ayudarnos en desentrañar las formas de sociabilidad femenina ${ }^{17}$.

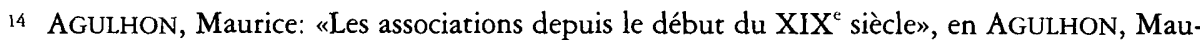
rice y BoDIguel, Maryvonne: Les Associations au village, Le Paradou, Actes Sud (Bibliothèque des ruralistes), 1981, p. 11.

15 AgUlHon, Maurice: «Classes ouvrières et sociabilité avant 1848 », Histoire vagabonde, vol. I (Ethnologie et politique dans la France contemporaine), Paris, Gallimard, 1988, p. 61, publicado primero en inglés ("Working class and sociability in France before 1848», en THANE, Pat, CrossiCK, Geoffrey y FLOYD, Roderick (Eds.): The power of the past. Essays for Eric Hobsbaum, Cambridge, Cambridge University Press, 1984, pp. 37-66). El texto ha sido posteriormente traducido al español en la revista Historia Social, Valencia, $\mathrm{n}^{\circ} 12$, Invierno 1992, pp. 141-166 («Clase obrera y sociabilidad antes de 1848»).

16 Bussy-Genevois, Danièle, Guereña, Jean-Louis y RALLe, Michel (Eds.): Fêtes, sociabilités, politique dans l'Espagne contemporaine, Parte monográfica del Bulletin d'Histoire Contemporaine de l'Espagne, $\mathrm{n}^{\circ}$ 30-31, Diciembre de 1999-Junio de 2000 [2002], pp. 5-313.

17 BusSY-GenEvors, Danièle (Ed.): Les Espagnoles dans lbistoire. Une sociabilité démocratique (XIX $X X^{e}$ siècles), Saint-Denis, Presses Universitaires de Vincennes (Culture et Société), 2002, 287 p.
}

Hispania, LXIII/2, núm. 214 (2003) 409-414 
Y si nos ceñimos en este número a la época contemporánea, cabe recordar finalmente que por supuesto la sociabilidad no nace en el siglo XIX sino que adquiere entonces nueva visibilidad y extraordinario desarrollo y diversidad en relación con nuevas demandas de ocio y de cultura.

Apuntemos finalmente la necesidad de realizar enfoques comparativos, dentro de España naturalmente y también con otros conjuntos culturales, principalmente europeos. Esperamos que esta sección monográfica contribuya a ello, para poder disponer de una visión transversal de las sociabilidades y establecer las relaciones existentes entre sociabilidad cultural, sociabilidad política y sociabilidad recreativa.

Hispania, LXIII/2, núm. 214 (2003) 409-414 